\title{
Water thermophoresis in carbon nanotubes: the interplay between thermophoretic and
} friction forces

Oyarzua, Elton; Walther, Jens Honore; Zambrano, Harvey A.

\author{
Published in: \\ Physical Chemistry Chemical Physics
}

Link to article, DOI:

$10.1039 / \mathrm{c} 7 \mathrm{cp} 05749 \mathrm{k}$

Publication date:

2018

Document Version

Peer reviewed version

Link back to DTU Orbit

Citation (APA):

Oyarzua, E., Walther, J. H., \& Zambrano, H. A. (2018). Water thermophoresis in carbon nanotubes: the interplay between thermophoretic and friction forces. Physical Chemistry Chemical Physics, 20, 3672-3677 .

https://doi.org/10.1039/c7cp05749k

\section{General rights}

Copyright and moral rights for the publications made accessible in the public portal are retained by the authors and/or other copyright owners and it is a condition of accessing publications that users recognise and abide by the legal requirements associated with these rights.

- Users may download and print one copy of any publication from the public portal for the purpose of private study or research.

- You may not further distribute the material or use it for any profit-making activity or commercial gain

- You may freely distribute the URL identifying the publication in the public portal 


\title{
Water thermophoresis in Carbon Nanotubes: the
} interplay between thermophoretic and friction

\section{forces}

\author{
Elton Oyarzua, ${ }^{\dagger}$ Jens Honore Walther, ${ }^{\ddagger}$ and Harvey A. Zambrano ${ }^{*, \dagger}$ \\ $\dagger$ Department of Chemical Engineering, Universidad de Concepcion, Concepcion, Chile \\ $\ddagger$ Department of Mechanical Engineering, Technical University of Denmark, DK-2800 Kgs. \\ Lyngby, Denmark \\ ฯ Computational Science and Engineering Laboratory, Department of Mechanical and \\ Process Engineering, ETH Zurich, CH-8092 Zurich, Switzerland \\ E-mail: harveyzambrano@udec.cl \\ Phone: +56 (0)41 2201468
}

\begin{abstract}
Thermophoresis is the phenomenon wherein particles experience a net drift induced by a thermal gradient. In this work, molecular dynamics simulations are conducted to study with atomistic detail the thermophoresis of water nanodroplets inside carbon nanotubes (CNTs) and its interplay with the retarding liquid-solid friction. Different applied temperatures, thermal gradients, and droplet sizes are used to reveal the dynamics of the two kinetic regimes of the thermophoretic motion in CNTs. The results indicate that during the droplet motion, the thermophoretic force is independent of the velocity of the droplet, whereas the magnitude of the retarding friction
\end{abstract}


force exhibits a linear dependence. In fact, in the initial regime the magnitude of the friction force increases linearly with the droplet velocity, until the thermophoretic force is balanced by the friction force as the droplet reaches its terminal velocity in the final regime. In addition, an increasing magnitude of the thermophoretic force is found for longer water droplets. These findings provide a deeper understanding of liquid transport driven by temperature gradients in nanoconfined geometries where liquid-solid interfaces govern fluidics.

\section{Introduction}

Thermophoresis is a directed motion of particles caused by the presence of an externally imposed thermal gradient. In particular, thermophoretic movement of a particle suspended in a molecular media is the consequence of a thermally rectified Brownian motion ${ }^{1,2}$ : molecules in the hotter region of the media collide with the particle, transferring a greater momenta as compared to the molecules in the colder regions. During more than a century, theoretical and experimental studies have been conducted to understand the factors governing the termophoretic transport and elucidate its complex underlying physics ${ }^{3-5}$. One of these pioneer investigations was made by Epstein in $1929^{6}$, who derived the first expressions for the thermophoretic force and velocity. Thereafter, motivated by its important practical consequences in the field of aerosol technology ${ }^{7}$, numerous investigations of thermophoresis of particles in gases have been conducted ${ }^{8-12}$. Thermophoresis of suspended particles in liquids was first studied by McNab and Meisen ${ }^{13}$, who found thermophoretic motion of a particle to be independent of the particle size. More recently, and motivated for potential applications of nanofluid solutions, this phenomenon has also been studied in dispersions of nanoscale particles immersed in fluids ${ }^{14-16}$. Thermophoresis is fundamentally related with the phenomenon of thermodiffusion in liquid solutions ${ }^{4,17}$, also called the Ludwig-Soret effect ${ }^{4,12}$ after Carl Ludwig ${ }^{18}$ and Charles Soret ${ }^{19}$ who independently studied this phenomenon in the 19 th century. 

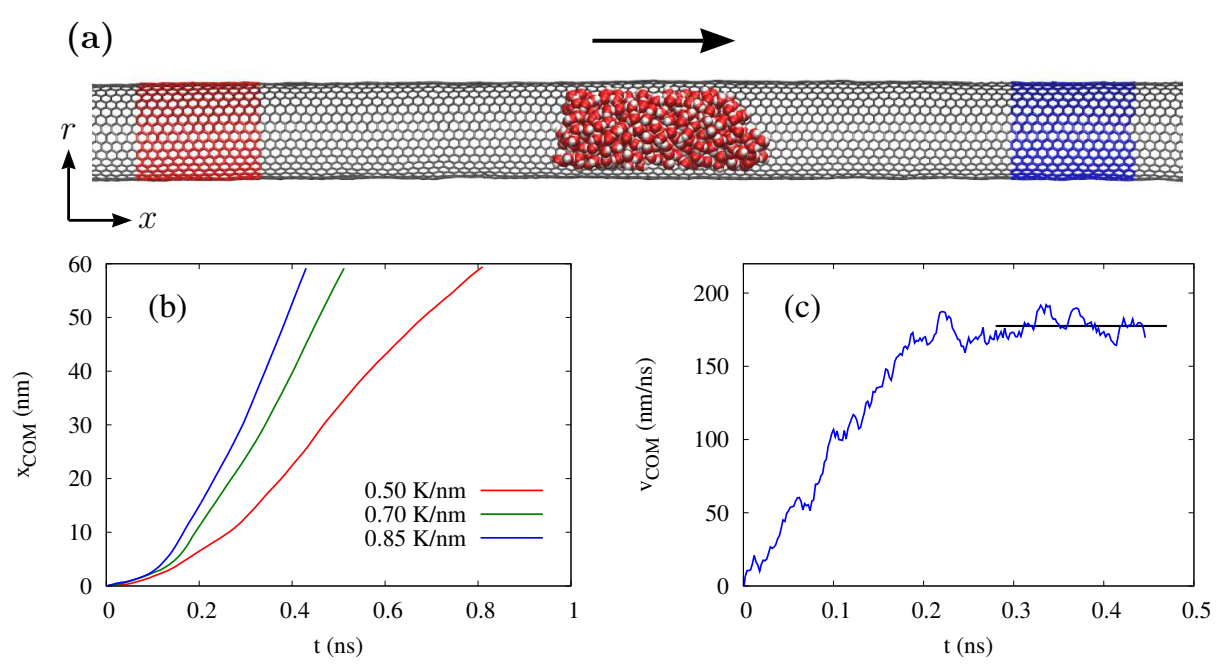

Figure 1: (a) Scheme of the studied system. The droplet inside the CNT moves from the high temperature side towards the cooler side of the CNT. The thermostat is applied directly to the carbon atoms, the red zone represents the high temperature section while the blue zone represents the low temperature section. (b) Time evolution of the center of mass position of the droplet consisting of 800 water molecules under different imposed thermal gradients. (c) History of the velocity of the center of mass of the droplet consisting of 400 water molecules under a imposed thermal gradient of $0.50 \mathrm{~K} / \mathrm{nm}$. The solid line depicts the constant velocity regime.

Nowadays, the advent of extremely accurate nanofabrication techniques has led to envision novel integrated nanofluidic devices wherein the functional stations are connected by nanoconduits ${ }^{20-22}$. In this context, thermophoresis may play a critical role as an enabling technology for achieving controlled transport of fluids in nanoscale devices. Recently, carbon nanotubes ${ }^{23}$ (CNTs) have emerged as ideal conduits for ultra efficient water transport $^{24-27}$ due to their molecularly smooth graphitic walls ${ }^{24}$, well controlled diameter, spatial nanoconfinement and outstanding thermal and mechanical properties ${ }^{25,28}$. Hence, the thermophoretic transport of fluids through the inner-core of CNTs has considerable technological and scientific implications. In fact, the practical use of thermophoresis in future CNT-based nanofluidic devices requires the feasibility of achieving predictable and controllable thermophoretic water transport inside CNTs. To date, despite the important efforts that have been devoted to investigate thermophoresis inside and outside $\mathrm{CNTs}^{29-41}$, the interplay between applied thermal gradient, thermophoretic velocity, solid-liquid friction ${ }^{42-45}$ 
and thermophoretic force ${ }^{30,33,36}$ in a water/CNT system, has not been studied. In this letter, we present an atomistic study of the kinetics associated with thermophoresis of water nanodroplets confined inside single wall carbon nanotubes and its interplay with the solid-liquid friction force.

\section{Methodology}

To carry out this investigation, we employ both constrained and unconstrained Molecular Dynamics (MD) simulations of nanodroplets confined in CNTs which are subjected to a constant axial thermal gradient. The axial thermal gradient is applied by coupling two Berendsen thermostats ${ }^{46}$ at different temperatures to the carbon atoms at the respective ends of the CNT cf. Fig. 1a. As demonstrated in previous studies, a Berendsen thermostat is suitable for imposing proper nonequilibrium conditions in $\mathrm{CNTs}^{47,48}$, and also exhibit correct mechanical responses at relatively constant temperature during CNT compression ${ }^{49}$. The MD simulations were performed using the parallel MD package FASTTUBE ${ }^{29,40,50,51}$. The equations of motion are integrated in time using the leapfrog scheme with a time step of $2 \mathrm{fs}$. Periodic boundary conditions is considered in the direction parallel to the CNT axis and with free space conditions in the normal directions. The carbon-carbon intramolecular interactions of the CNT are described by a Morse bond, a harmonic cosine of the bending angle, and a torsion potential $29,30,40,50$. The water is modeled using the rigid SPC/E water model ${ }^{52}$ with the $\mathrm{O}-\mathrm{H}$ bond and the $\mathrm{H}-\mathrm{O}-\mathrm{H}$ angle constrained using the SHAKE algorithm. The water-CNT interactions are described by a 12-6 LJ potential calibrated to reproduce a water contact angle of $81^{\circ}$ as described by Werder et al. ${ }^{53}$ and Zambrano et al. ${ }^{40}$. The van der Waals and Coulomb interactions are truncated at $1.0 \mathrm{~nm}$ with the Coulomb potential smoothly truncated to ensure energy conservation ${ }^{40,50}$. The MD package and the force fields have been extensively validated in previous studies of thermophoresis ${ }^{29,30,36,40,53,54}$. For details of the potentials and setup of the simulations, we refer the reader to Zambrano et al. ${ }^{40}$. 
In the unconstrained MD simulations of thermophoresis ${ }^{29,33-37,40,41}$, a constant thermal gradient is applied along the axis of the CNT. The imposed thermal gradient induces a thermophoretic motion of the confined nanodroplet in the opposite direction of the thermal gradient. Moreover, in line with the results presented by Shiomi and Maruyama ${ }^{41}$, two regimes in the movement of the nanodroplet are observed: first an acceleration regime wherein the thermophoretic force is larger than the friction force, and then, a constant velocity regime, wherein the thermophoretic force balances the friction force and the droplet reaches its terminal velocity. Furthermore, to gain insight in the kinetics of the droplet motion, constrained MD simulations are conducted. This set of simulations consists of setting the center of mass velocity $\left(v_{\text {com }}\right)$ every time step to a target value, forcing the droplet to move with a constrained velocity without affecting the velocity distribution of the molecules in the droplet. Constrained MD simulations are performed with and without imposed thermal gradients. The MD simulations with constrained velocity were introduced by Schoen et al. ${ }^{29}$ and then, in a similar context, reported by Zambrano et al. ${ }^{36}$. Further details of this technique are provided in the Supporting Information.

\section{Results and discussion}
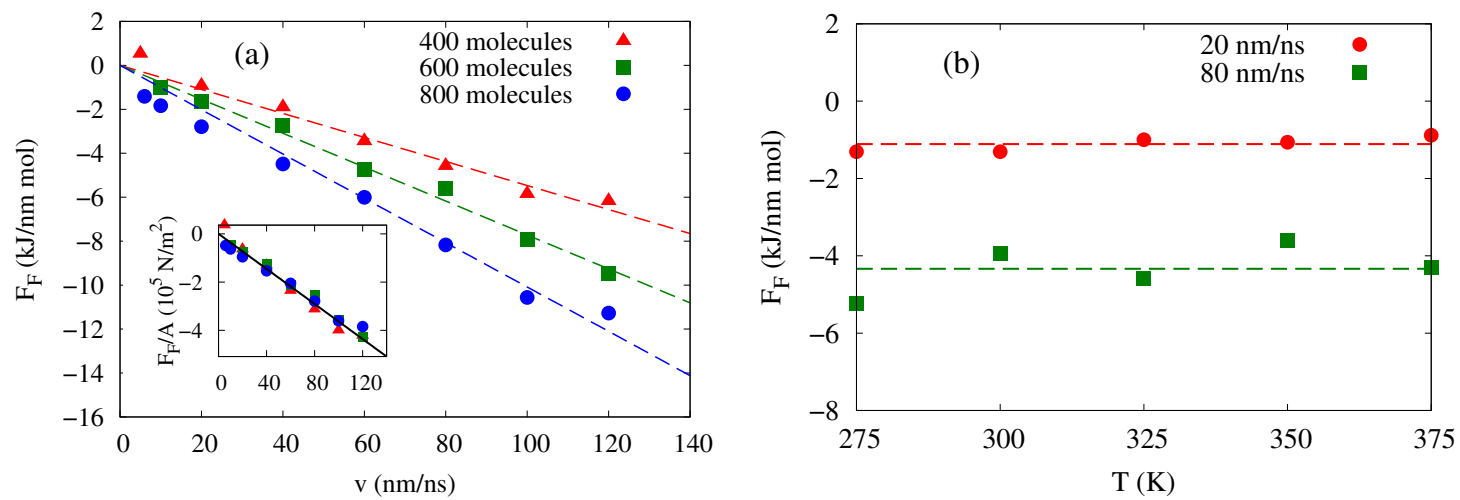

Figure 2: (a) Friction force as a function of the velocity of center of mass $\left(v_{\text {com }}\right)$ for droplet sizes $400(\boldsymbol{\Delta}), 600(\mathbf{\square})$ and $800(\bullet)$ water molecules at $325 \mathrm{~K}$. The dashed lines are fits to the data. Inset: Friction forces divided by the respective solid-liquid contact area. (b) Friction force a function of temperature with imposed $v_{\text {com }}$ of $20 \mathrm{~nm} / \mathrm{ns}$ and $80 \mathrm{~nm} / \mathrm{ns}$ with a droplet of 400 water molecules. The dashed lines are fits to the data. 
In this study, an armchair CNT with chirality $(17,17)$ and length of $75 \mathrm{~nm}$ is considered for all cases. Thermal gradients of $0.2,0.5,0.7$ and $0.85 \mathrm{~K} / \mathrm{nm}$ are imposed along the axis of the CNT wherein water nanodroplets of different sizes are confined. The nanodroplets confined in the CNTs consist of 400, 600 and 800 water molecules. A schematic of the system is shown in Fig. 1a. From the atomistic trajectories, the temporal evolution of both position and velocity of the center of mass of the droplets are extracted. In line with previous studies $^{29,36,40,41}$, a directed displacement of the droplet is observed from the high temperature zone towards the low temperature zone. Moreover, for all cases we observe higher $v_{\text {com }}$ as higher thermal gradients are imposed along the CNT cf. Fig. 1b. Furthermore, as shown in Fig. 1c, during the droplet displacement two dynamic regimes can be identified: first a regime with increasing velocity in time and subsequently, a constant velocity regime as depicted by the solid line. We notice that in this set of simulations, the only forces acting on the droplet are the thermophoretic force $\left(F_{T}\right)$ which is the force exerted by the thermal gradient, and the retarding friction force $\left(F_{F}\right)$ acting at the solid-liquid interface, hence, the resulting force is

$$
F_{N}=m \cdot a=F_{T}-F_{F}
$$

where $F_{N}$ is the net force, $m$ is the mass of the droplet and $a$ is the instantaneous acceleration. Figure $1 \mathrm{c}$ infers that within the first regime the magnitude of the thermophoretic force acting on the droplet is higher than the magnitude of the friction force, conversely, during the constant velocity regime, the retarding friction force balances the thermophoretic force, i.e., the droplet reaches its maximum velocity at zero net force. The behavior exhibited by the droplet motion suggests that the instantaneous magnitude of the friction force and/or the thermophoretic force must be dependent on the instantaneous velocity of the droplet. In order to analyze the relationship between the droplet speed and the thermophoretic and friction forces, sets of isothermal and non-isothermal MD simulations are performed at different constrained velocities. From the constrained simulations with an imposed thermal gradient, the instantaneous net force $\left(F_{N}\right)$, i.e., the force instantaneously accelerating the 
droplet is computed from the simulations, similar to the analysis performed by Schoen et al. ${ }^{29}$ and Zambrano et al. ${ }^{36}$. Furthermore, the instantaneous retarding friction force $\left(F_{F}\right)$ is computed from the constrained simulations at constant temperature. The thermophoretic force $\left(F_{T}\right)$, which is the force exerted on the droplet by the imposed thermal gradient, is calculated by adding the friction force $\left(F_{F}\right)$ to the net force $\left(F_{N}\right)$ at the respective velocity, according Eq. (1).

We notice that the center of mass velocity $\left(v_{\text {com }}\right)$ of the droplet is assumed to be equivalent to the slip velocity considering the ultra high slippage inherent in the water-CNT interface, in line with Falk et al. ${ }^{42}$. In fact, previous investigations ${ }^{27,55,56}$ reported water slip length over $75 \mathrm{~nm}$ for a CNT of $2 \mathrm{~nm}$ in diameter, leading to a plug-like velocity profile for water confined in $\mathrm{CNTs}^{40,45}$. Furthermore, Chen et al. ${ }^{57}$ found that water droplets spontaneously slip inside CNTs owing to thermal fluctuations of water at room temperature.

The friction force as a function of the constrained center of mass velocity is shown in Fig. 2a. In line with the previous studies ${ }^{42,43,58}$, the magnitude of the friction force increases for higher velocities of the droplet. Indeed, the magnitude of the friction force is linearly proportional to the droplet velocity. Note that the negative values in the magnitude of the friction force are due to the force direction opposite to the displacement of the nanodroplet. In Fig. 2a, each point is computed from the atomic trajectories obtained from independent $3 \mathrm{~ns}$ MD simulations at constant temperature, the red, green and blue dashed lines are fits to the data assuming $F_{F}=0$ for $v=0^{57}$. Moreover, the dashed lines in Fig. 2a. display different slopes because each nanodroplet (400, 600 and 800 molecules) has different liquid-solid contact areas. In fact, if the magnitudes of the computed friction forces presented in Fig. 2a are divided by the respective contact area, all the data converge to a single slope, as shown in the inset of Fig. 2a, where the magnitude of the slope corresponds to a friction coefficient of $3631 \mathrm{Ns} / \mathrm{m}^{3}$. Moreover, as the temperature imposed along the CNT is systematically varied in each isothermal simulation, the friction force is found to be independent of the imposed temperature cf. Fig. 2b. Thus, in non-isothermal simulations we assume that the magnitude 
of the friction force does not change as different thermal gradient are imposed along the CNT.
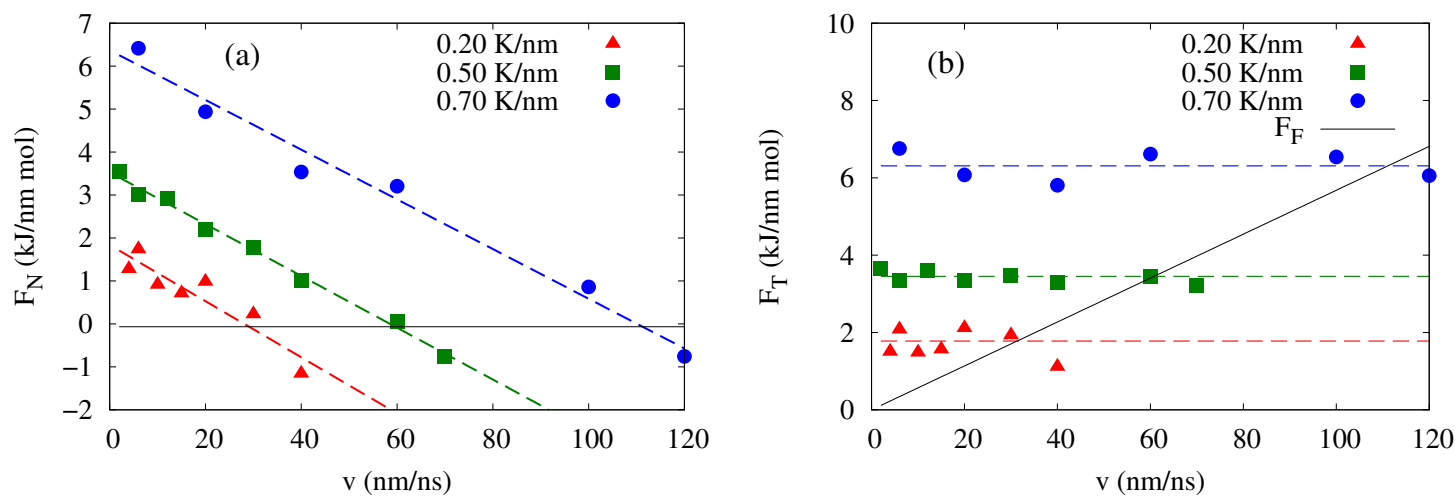

Figure 3: Net force and thermophoretic force of the 400 water molecules case under thermal gradients of $0.20 \mathrm{~K} / \mathrm{nm}(\boldsymbol{\Delta}) 0.50 \mathrm{~K} / \mathrm{nm}(\boldsymbol{\nabla})$ and $0.70 \mathrm{~K} / \mathrm{nm}(\bullet)$. (a) Net force as a function of the velocity of center of mass. The solid black line is a guide for $F_{N}=0$. (b) Thermophoretic force as a function of the velocity of center of mass. The dashed lines are fits to the data. The solid black line is the absolute value of the friction force for the 400 water molecules case (Fig 2.a).

The net force $\left(F_{N}\right)$ acting on the nanodroplets is computed from constrained MD simulations with imposed thermal gradients. Constant velocities $\left(v_{\mathrm{com}}\right)$ ranging from $3 \mathrm{~m} / \mathrm{s}$ to $120 \mathrm{~m} / \mathrm{s}$ are imposed to the center of mass of the droplet while thermal gradients of 0.20 , 0.50 and $0.70 \mathrm{~K} / \mathrm{nm}$ are applied along the axis of the CNT. For the case of a water droplet of 400 molecules, the net force as a function of the imposed velocity $\left(v_{\text {com }}\right)$ is depicted in Fig. 3a. For all the imposed thermal gradients, we observe that the instantaneous net force decreases linearly with the imposed velocity $\left(v_{\text {com }}\right)$, starting from a positive value at zero velocity and decreasing until it vanishes when the droplet achieves its terminal velocity. Moreover, it should be noted in Fig. 3a that the slope of the blue, green and red dashed lines is equivalent to the slope for the corresponding dashed red line in Fig. 2a, which is the friction coefficient times the solid-liquid contact area. The dashed red line in Fig. 2a shows the friction force versus velocity for the same droplet consisting of 400 water molecules, therefore, the decrease in the net force is due to the growth of the friction force with higher velocities. Since $F_{N}=m \cdot a$, the thermophoretic motion of the droplet is associated with 
a decreasing acceleration during the first regime (see Support Info. Figure S4), before the terminal velocity is reached, showing no evidence of a constant acceleration regime during the thermophoretic motion as inferred by previous authors ${ }^{41,59}$.

From the computed friction and the instantaneous net forces, the thermophoretic force is calculated. In Fig. 3b the thermophoretic force for the 400 water molecules case is presented, wherein each point is obtained from the sum of the net force $\left(F_{N}\right)$ computed from the constrained simulations (Fig. 3a) and the friction force $\left(F_{F}\right)$ (Fig. 2a) at the corresponding velocity, according to Eq. (1). As depicted in Fig. 3b, the thermophoretic force displays not dependency on the droplet velocity, i.e., the droplet is subjected to a constant thermophoretic force during the motion. Moreover, Fig. 3b shows that for higher imposed thermal gradients, the thermophoretic force increases as observed in previous studies ${ }^{29,33,36,41}$. We note that the relation between velocity and thermophoretic force has been studied for systems consisting of motile coaxial $\mathrm{CNTs}^{36}$ wherein a decreasing thermophoetic force has been observed for higher velocities of the inner CNT. Conversely, in the present study for a solid-liquid interface, we find that the thermophoretic force is not velocity dependent while the friction force increases linearly with the droplet speed.
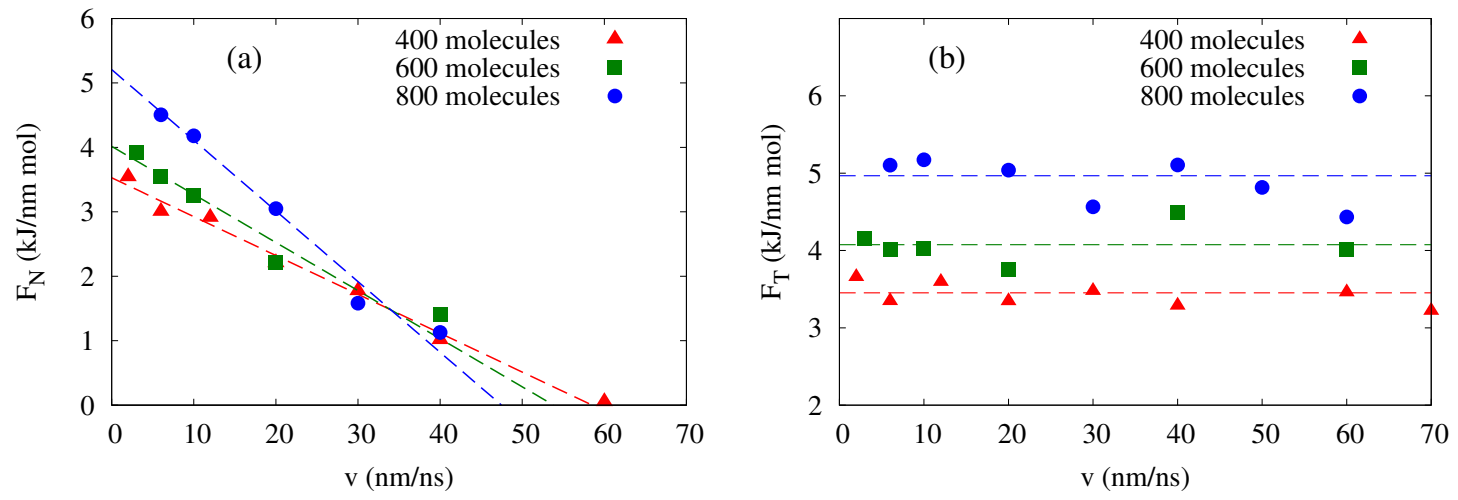

Figure 4: Net force and thermophoretic force for droplet sizes of $400(\mathbf{\Delta}), 600(\boldsymbol{\square})$ and $800(\bullet)$ water molecules under an applied thermal gradient of $0.50 \mathrm{~K} / \mathrm{nm}$. (a) Computed Net force as a function of the velocity of the center of mass. The dashed lines are fits to the data. (b) Thermophoretic force as a function of the velocity of center of mass. The dashed lines are fits to the data.

It is also interesting to evaluate the effect of the size of the droplets on the thermophoretic 
and net force. The net force as a function of the constrained center of mass velocity is shown in Fig. 4a for droplets consisting of 400 (red triangles), 600 (green squares) and 800 (blue circles) molecules under an imposed thermal gradient of $0.50 \mathrm{~K} / \mathrm{nm}$. The dashed lines in Fig. 4a are linear fits for each case, with the slopes depicting the friction coefficient times the respective solid-liquid contact area. Note that the solid-liquid contact area is larger for droplets with higher number of water molecules. Therefore, Fig. 4a indicates that all the nanodroplets move with a decelerated motion, slowed down with a rate that is directly proportional to the size of the particular nanodroplet (See Fig. 2a), i.e., at the same instantaneous speed, droplets with a larger solid-liquid contact area experience a higher retarding friction. Indeed, as shown in Fig. 4a for an imposed thermal gradient of $0.50 \mathrm{~K} / \mathrm{nm}$, the magnitude of the terminal velocity is higher for droplets with smaller number of water molecules. The same behavior is observed for different imposed thermal gradients as shown in Supporting Info. Figure S5. Furthermore, the thermophoretic force is computed from the net force values presented in Fig. 4a. In Fig. 4b, for an imposed thermal gradient of $0.50 \mathrm{~K} / \mathrm{nm}$, higher thermophoretic forces are computed for longer water droplets, which shows that the magnitude of the thermophoretic force is directly related to the droplet size (or the droplet length due to the constant CNT cross-section). It should be noted that there is no consensus about the relation between the thermophoretic force and the size of the motile particle. For example, the thermophoretic force has been found to be dependent of particle size for distributions of particles in gas media ${ }^{60,61}$ and for particles inside $\mathrm{CNTs}^{62}$. On the other hand, a non size dependence of the thermophoretic force has been found for solid particles in liquid media ${ }^{13}$, oil droplets in liquid mixtures ${ }^{63}$ and double-walled CNTs ${ }^{33}$. Here, to gain insight into the relationship between the thermophoretic force and the particular size of the water nanodroplet, Fig. 5 depicts the thermophoretic force as a function of the thermal gradient for droplets consisting of 400, 600 and 800 water molecules. The results indicate that the magnitude of the thermophoretic force acting on the droplet is directly related to both the magnitude of the imposed thermal gradient and the particular length of the droplet. 
Therefore, in line with previous studies ${ }^{30,31}$, we infer that the thermophoretic force acting on the droplet is generated by the net current of phonons induced by the imposed thermal gradient, i.e., the rectified thermal vibrations of the carbon atoms provide the effective force to drive the water droplet through the CNT. Nevertheless, we notice further investigation is required to find the precise relationship between the interfacial phonon scattering dynamics and the thermophoretic force acting on nanodroplets confined in CNTs. We believe that this investigation provides deeper understanding of liquid transport driven by temperature gradients in nanodevices and will be useful for the development of nanofluidic applications.

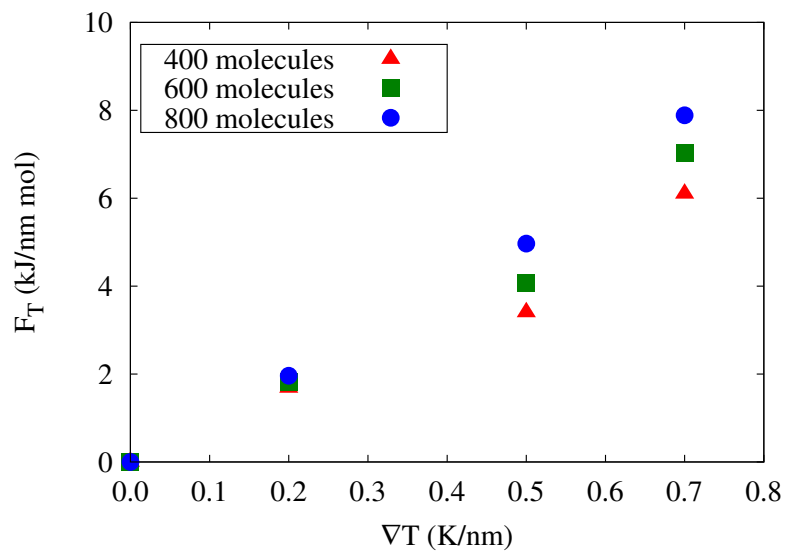

Figure 5: Thermophoretic force as a function of the thermal gradient for droplet sizes of $400(\boldsymbol{\Lambda}), 600(\mathbf{\bullet})$ and $800(\bullet)$ water molecules.

\section{Conclusions}

In summary, by using molecular dynamics we studied in detail the interplay between thermophoretic and friction forces which govern the thermophoretic transport of water nanodroplets through CNTs. The results indicate that the thermophoretic force is not velocity dependent while the friction force increases linearly with the droplet speed. Moreover, we find that the magnitude of the thermophoretic force is determined by the imposed thermal gradient and the particular length of the droplet. In general, the thermophoretic motion of a nanodroplet exhibits two kinetic regimes: (i) a initial regime wherein the droplet moves with decreasing acceleration, i.e., the friction force is linearly proportional to the droplet velocity 
whereas the thermophoretic force has a constant value defined by the thermal gradient and the droplet length, and (ii) a subsequent regime wherein the droplet moves at constant velocity due to balance between the thermophoretic force and the retarding friction force. The results presented in this letter contribute to gain insight in the transport of liquids driven by thermal gradients and have practical implications for the design of CNT-based nanofluidic devices.

\section{Acknowledgement}

E. Oyarzua thanks financial support from CONICYT scholarship no. 21140427. This research was partially funded by CONICYT under FONDECYT project No 11130559 . We also thank the partial financial support from the University of Concepcion under VRID project no. 21496651. The authors received computational support from the Department of Physics at the Technical University of Denmark. Furthermore, the authors wish to acknowledge professor Constantine M. Megaridis for valuable scientific discussions.

\section{References}

1. Michaelides, E. E. Brownian movement and thermophoresis of nanoparticles in liquids. Int. J. Heat Mass Transfer 2015, 81, 179-187.

2. Plyukhin, A. V. Thermophoresis as persistent random walk. Phys. Lett. A 2009, 373, $2122-2124$.

3. Maxwell, J. C. On stresses in rarified gases arising from inequalities of temperature. Philosophical Transactions of the royal society of London 1879, 170, 231-256.

4. Rahman, M.; Saghir, M. Thermodiffusion or Soret effect: Historical review. Int. J. Heat Mass Transfer 2014, 73, 693-705. 
5. Srinivasan, S.; Saghir, M. Z. Experimental approaches to study thermodiffusion: A review. Internat. J. Thermal Sci. 2011, 50, 1125-1137.

6. Epstein, P. S. Zur theorie des radiometers. Zeitschrift für Physik 1929, 54, 537-563.

7. Zheng, F. Thermophoresis of spherical and non-spherical particles: a review of theories and experiments. Adv. Coll. Interf. Sci. 2002, 97, 255-278.

8. Brock, J. R. On the theory of thermal forces acting on aerosol particles. J. Coll. Sci. 1962, 17, 768-780.

9. Talbot, L.; Cheng, R.; Schefer, R.; Willis, D. Thermophoresis of particles in a heated boundary layer. J. Fluid Mech. 1980, 101, 737-758.

10. Derjaguin, B.; Yalamov, Y. Theory of thermophoresis of large aerosol particles. J. Coll. Sci. 1965, 20, 555-570.

11. Li, W.; James Davis, E. The effects of gas and particle properties on thermophoresis. $J$. Aerosol Sci. 1995, 26, 1085-1099.

12. Debbasch, F.; Rivet, J. P. The LudwigSoret effect and stochastic processes. J. Chem. Thermodynamics 2011, 43, 300-306.

13. McNab, G.; Meisen, A. Thermophoresis in liquids. J. Coll. Interface Sci. 1973, 44, $339-346$.

14. Haddad, Z.; Abu-Nada, E.; Oztop, H. F.; Mataoui, A. Natural convection in nanofluids: Are the thermophoresis and Brownian motion effects significant in nanofluid heat transfer enhancement? Internat. J. Thermal Sci. 2012, 57, 152-162.

15. Buongiorno, J. Convective transport in nanofluids. J. Heat Transfer 2006, 128, 240-250.

16. Martin, A.; Bou-Ali, M. M. Determination of thermal diffusion coefficient of nanofluid: Fullerene-toluene. Comptes Rendus Mécanique 2011, 339, 329-334. 
17. Wang, J.; Li, Z. Negative thermophoresis of nanoparticles in the free molecular regime. Phys. Rev. E 2012, 86, 011201.

18. Ludwig, C. Diffusion between unequally heated regions of initially uniform solutions. Sitzber. Bayer Akad. Wiss. Wien 1856, 20, 539.

19. Soret, C. Sur letat dequilibre que prend au point de vue de sa concentration une dissolution saaline primitivement homogene dont deux parties sont portees a des temperatures differentes. Ann. Chim. Phys. 1881, 22, 293-297.

20. Geelhoed, P.; Lindken, R.; Westerweel, J. Thermophoretic separation in microfluidics. Chemical Engineering Research and Design 2006, 84, 370-373.

21. Thamdrup, L. H.; Larsen, N. B.; Kristensen, A. Light-Induced Local Heating for Thermophoretic Manipulation of DNA in Polymer Micro- and Nanochannels. Nano Lett. 2010, 10, 826-832.

22. Zheng, Y.; Liu, H.; Wang, Y.; Zhu, C.; Wang, S.; Cao, J.; Zhu, S. Accumulating microparticles and direct-writing micropatterns using a continuous-wave laser-induced vapor bubble. Lab on a Chip 2011, 11, 3816-3820.

23. Iijima, S. Helical microtubules of graphitic carbon. NATURE 1991, 354, 56-58.

24. Joseph, S.; Aluru, N. R. Hierarchical multiscale simulation of electrokinetic transport in silica nanochannels a the point of zero charge. Langmuir 2006, 22, 9041-9051.

25. Berber, S.; Kwon, Y.-K.; Tománek, D. Unusually high thermal conductivity of carbon nanotubes. Phys. Rev. Lett. 2000, 84, 4613.

26. Majumder, M.; Chopra, N.; Andrews, R.; Hinds, B. J. Nanoscale hydrodynamics: enhanced flow in carbon nanotubes. NATURE 2005, 438, 44-44. 
27. Holt, J. K.; Park, H. G.; Wang, Y.; Stadermann, M.; Artyukhin, A. B.; Grigoropoulos, C. P.; Noy, A.; Bakajin, O. Fast mass transport through sub-2-nanometer carbon nanotubes. Science 2006, 312, 1034-1037.

28. Treacy, M. J.; Ebbesen, T.; Gibson, J. Exceptionally high Young's modulus observed for individual carbon nanotubes. NATURE 1996, 381, 678.

29. Schoen, P. A.; Walther, J. H.; Arcidiacono, S.; Poulikakos, D.; Koumoutsakos, P. Nanoparticle traffic on helical tracks: thermophoretic mass transport through carbon nanotubes. Nano Lett. 2006, 6, 1910-1917.

30. Schoen, P. A.; Walther, J. H.; Poulikakos, D.; Koumoutsakos, P. Phonon assisted thermophoretic motion of gold nanoparticles inside carbon nanotubes. Appl. Phys. Lett. 2007, 90, 253116.

31. Barreiro, A.; Rurali, R.; Hernandez, E. R.; Moser, J.; Pichler, T.; Forro, L.; Bachtold, A. Subnanometer motion of cargoes driven by thermal gradients along carbon nanotubes. Science 2008, 320, 775-778.

32. Rurali, R.; Hernandez, E. Thermally induced directed motion of fullerene clusters encapsulated in carbon nanotubes. Chem. Phys. Lett. 2010, 497, 62-65.

33. Hou, Q.-W.; Cao, B.-Y.; Guo, Z.-Y. Thermal gradient induced actuation in double-walled carbon nanotubes. Nanotechnol. 2009, 20, 495503.

34. Prasad, M. V.; Bhattacharya, B. Phonon Scattering Dynamics of Thermophoretic Motion in Carbon Nanotube Oscillators. Nano Lett. 2016, 16, 2174-2180.

35. Chen, J.; Gao, Y.; Wang, C.; Zhang, R.; Zhao, H.; Fang, H. Impeded Mass Transportation Due to Defects in Thermally Driven Nanotube Nanomotor. J. Phys. Chem. C 2015, 119, 17362-17368. 
36. Zambrano, H. A.; Walther, J. H.; Jaffe, R. L. Thermally driven molecular linear motors: a molecular dynamics study. J. Chem. Phys. 2009, 131, 241104.

37. Guo, Z.; Chang, T.; Guo, X.; Gao, H. Mechanics of thermophoretic and thermally induced edge forces in carbon nanotube nanodevices. J. Mech. Phys. Solids 2012, 60, $1676-1687$.

38. Santamaría-Holek, I.; Reguera, D.; Rubi, J. Carbon-nanotube-based motor driven by a thermal gradient. J. Phys. Chem. C 2013, 117, 3109-3113.

39. Wei, N.; Wang, H.-Q.; Zheng, J.-C. Nanoparticle manipulation by thermal gradient. Nanoscale Res. Lett. 2012, 7, 1-9.

40. Zambrano, H. A.; Walther, J. H.; Koumoutsakos, P.; Sbalzarini, I. F. Thermophoretic Motion of Water Nanodroplets Confined Inside Carbon Nanotubes. Nano Lett. 2009, 9, $66-71$.

41. Shiomi, J.; Maruyama, S. Water transport inside a single-walled carbon nanotube driven by a temperature gradient. Nanotechnology 2009, 20, 055708.

42. Falk, K.; Sedlmeier, F.; Joly, L.; Netz, R. R.; Bocquet, L. Molecular origin of fast water transport in carbon nanotube membranes: superlubricity versus curvature dependent friction. Nano Lett. 2010, 10, 4067-4073.

43. Ma, M. D.; Shen, L.; Sheridan, J.; Liu, J. Z.; Chen, C.; Zheng, Q. Friction of water slipping in carbon nanotubes. Phys. Rev. E 2011, 83, 036316.

44. Ma, M.; Grey, F.; Shen, L.; Urbakh, M.; Wu, S.; Liu, J. Z.; Liu, Y.; Zheng, Q. Water transport inside carbon nanotubes mediated by phonon-induced oscillating friction. Nature Nanotechnol. 2015, 10, 692-695.

45. Joseph, S.; Aluru, N. Why are carbon nanotubes fast transporters of water? Nano Lett. 2008, 8, 452-458. 
46. Berendsen, H. J. C.; Postma, J. P. M.; van Gunsteren, W. F.; DiNola, A.; Haak, J. R. Molecular dynamics with coupling to an external bath. J. Chem. Phys. 1984, 81, 36843684.

47. Berendsen, H. J. Simulating the physical world: hierarchical modeling from quantum mechanics to fluid dynamics; Cambridge University Press, 2007; pp 195-203.

48. Van Der Spoel, D.; Lindahl, E.; Hess, B.; Groenhof, G.; Mark, A. E.; Berendsen, H. J. GROMACS: fast, flexible, and free. J. Comput. Chem. 2005, 26, 1701-1718.

49. Heo, S.; Sinnott, S. B. Investigation of the influence of thermostat configurations on the mechanical properties of carbon nanotubes in molecular dynamics simulations. $J$. Nanosci. Nanotechnol. 2007, 7, 1518-1524.

50. Walther, J. H.; Jaffe, R.; Halicioglu, T.; Koumoutsakos, P. Carbon nanotubes in water: Structural characteristics and energetics. J. Phys. Chem. B 2001, 105, 9980-9987.

51. Walther, J. H.; Ritos, K.; Cruz-Chu, E. R.; Megaridis, C. M.; Koumoutsakos, P. Barriers to superfast water transport in carbon nanotube membranes. Nano Lett. 2013, 13, 19101914.

52. Berendsen, H. J. C.; Grigera, J. R.; Straatsma, T. P. The missing term in effective pair potentials. J. Phys. Chem. 1987, 91, 6269-6271.

53. Werder, T.; Walther, J. H.; Jaffe, R. L.; Halicioglu, T.; Koumoutsakos, P. On the water-graphite interaction for Use in MD simulations of graphite and carbon nanotubes. J. Phys. Chem. B 2003, 10\%, 1345-1352.

54. Werder, T.; Walther, J. H.; Jaffe, R.; Halicioglu, T.; Noca, F.; Koumoutsakos, P. Molecular dynamics simulations of contact angles of water droplets in carbon nanotubes. Nano Lett. 2001, 1, 697-702. 
55. Kannam, S. K.; Todd, B.; Hansen, J. S.; Daivis, P. J. How fast does water flow in carbon nanotubes? J. Chem. Phys. 2013, 138, 094701.

56. Thomas, J. A.; McGaughey, A. J. Reassessing fast water transport through carbon nanotubes. Nano Lett. 2008, 8, 2788-2793.

57. Chen, C.; Shen, L.; Ma, M.; Liu, J. Z.; Zheng, Q. Brownian motion-induced water slip inside carbon nanotubes. Microfluid. Nanofluid. 2014, 16, 305-313.

58. Dedkov, G. Friction on the nanoscale: new physical mechanisms. Materials Letters 1999, $38,360-366$.

59. Rajegowda, R.; Kannam, S. K.; Hartkamp, R.; Sathian, S. P. Thermophoretic transport of ionic liquid droplets in carbon nanotubes. Nanotechnol. 2017, 28, 155401.

60. He, C.; Ahmadi, G. Particle deposition with thermophoresis in laminar and turbulent duct flows. Aerosol Sci. Tech. 1998, 29, 525-546.

61. Wang, J.; Li, Z. Thermophoretic force on micro-and nanoparticles in dilute binary gas mixtures. Phys. Rev. E 2011, 84, 021201.

62. Zhao, J.; Huang, J.-Q.; Wei, F.; Zhu, J. Mass transportation mechanism in electric-biased carbon nanotubes. Nano Lett. 2010, 10, 4309-4315.

63. Vigolo, D.; Brambilla, G.; Piazza, R. Thermophoresis of microemulsion droplets: Size dependence of the Soret effect. Phys. Rev. E 2007, 75, 040401. 
${ }_{379}$ Graphical TOC Entry

380

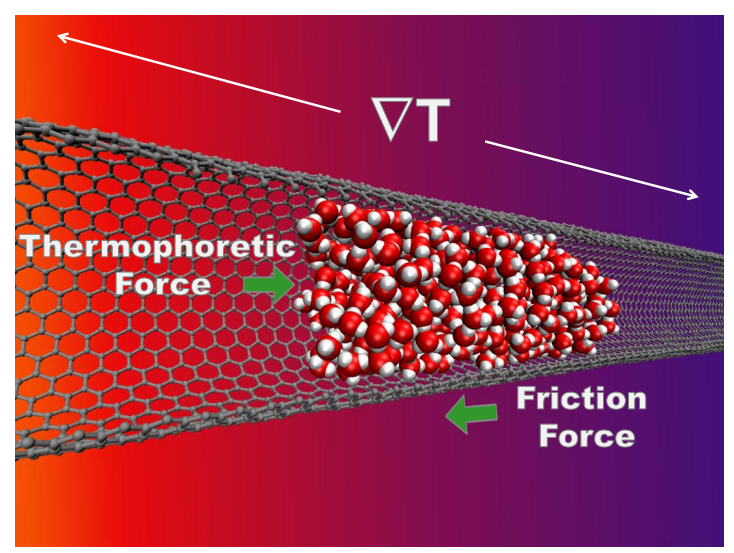

